Dietrich Manzey

\title{
Cognitive and Psychomotor Performance
}

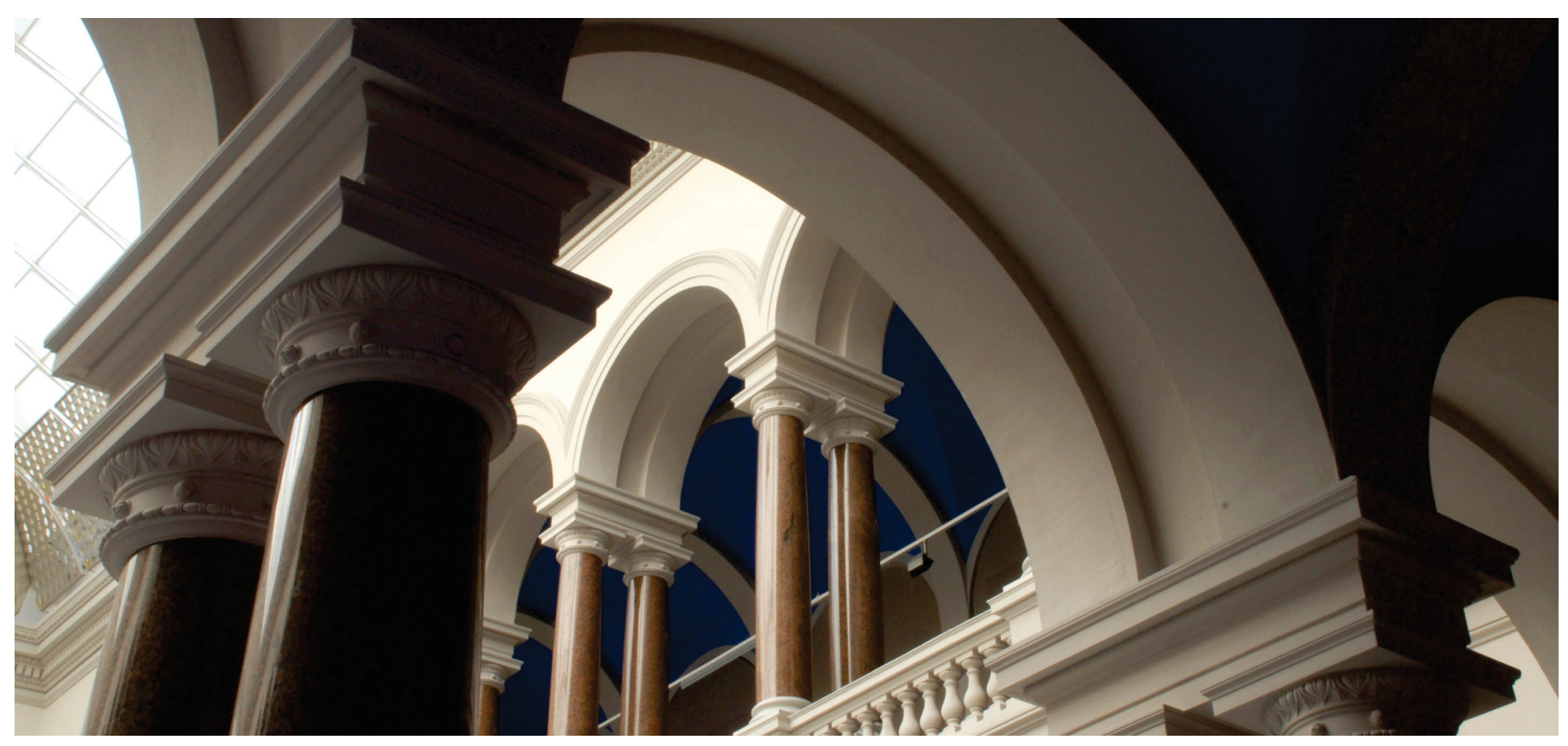

Manzey, D. (2017). Cognitive and Psychomotor Performance. Sensory Motor and Behavioral Research in Space, 47-58. https://doi.org/10.1007/978-3-319-68201-3_3 


\title{
Cognitive and Psychomotor Performance \\ Dietrich Manzey \\ Technische Universitaet Berlin \\ Institute of Psychology and Ergonomics
}

\begin{abstract}
During space flight, astronauts are exposed to a variety of stressors. Some of these stressors originate from the specific environmental conditions in space (e.g. microgravity, radiation). Others are more unspecific and originate from living and working as member of a small crew in a confined and isolated habitat (e.g. lack of privacy, social monotony). This chapter summarizes our current knowledge about the impact of these space flight-related stressors on cognitive and psychomotor performance of astronauts. It suggests that basic cognitive processes are highly resilient and remain as efficient in space as on Earth. Similarly, also processes of spatial imagery and object recognition do not seem to be affected much by the altered conditions in space. In contrast, considerable performance decrements have consistently been observed in different psychomotor tasks. These decrements seem to be caused by microgravity-induced changes of sensorimotor processes, at least during a transient period of primary adaptation to space. The available evidence pointing to impairments of executive functions and higher cognitive processes in space is less conclusive at this time.
\end{abstract}

Keywords Cognitive performance $\bullet$ Spatial cognition $\bullet$ Psychomotor performance $\bullet$ Spaceflight $\bullet$ Microgravity

\section{Introduction}

The motivation to study the impact of spaceflight on human cognitive and psychomotor performance has to main drivers. The first one reflects the general interest of basic neurocognitive research to better understand the underlying mechanisms of cognitive and sensorimotor processes and their plasticity and adaptability. During evolution, human beings have been shaped for optimal performance on Earth, that is, an environment which is characterized by specific natural constants, 
including, for example, gravity, and a 24 hours cycle of sunlight and darkness. However, when entering space, the environmental conditions are profoundly altered. This raises the basic question to what extent our brain functions are dependent on the usual conditions on Earth, how well brain functions can adapt to changes of these in space, and how this is reflected in the overt performance of astronauts.

The second driver which motivates the interest in cognitive and psychomotor performance of astronauts is a more practical one related to mission operations and crew support. During a space mission, astronauts have to cope with a large variety of tasks. These tasks span a large range from relatively simple housekeeping tasks to highly complex and sophisticated tasks related to the technical maintenance of the space station and the performance of experiments stemming from scientific areas as different as medicine, engineering, physics, material sciences, biology or radiology. Furthermore, specific challenges are provided by operations of the robot arm of the station and by so called extra-vehicular activities (EVA) which involve maintenance and repair tasks to be conducted at the outer shell of the space station (e.g. repair of solar panels) after leaving the station in space suits. All these different tasks put high demands on cognitive and psychomotor functions of astronauts (see for example a cognitive task analysis of robot arm operations provided by Morphew et al. 2001). Thus, maintaining a good functional performance state of astronauts and following possible performance impairments when planning and scheduling mission tasks is of paramount importance for mission success, and can be considered as a key issue of ensuring mission safety (Ellis 2000). This also requires detailed knowledge about what performance functions of astronauts are resilient in space and what performance functions might be prone to disturbances and impairments due to the typical stressors astronauts are exposed to during spaceflight.

\section{Stressors in Space}

Two different sorts of stressors in space can be distinguished which may affect the effectiveness or efficiency of cognitive and psychomotor functions (Kanas \& Manzey 2008). The first one includes stressors which are specific for the space environment, i.e. stressors we do not find in any 
environments on Earth. The most prominent stressors of this kind are microgravity, radiation and the altered dark-light cycle compared to the 24 hours day-night cycle on Earth. Although all of these space specific stressors can affect the performance of astronauts, microgravity is perhaps the most important one with respect to human performance functions. The almost eliminated impact of gravitational force in an orbiting spacecraft has been shown to lead to several neurophysiological changes (Newberg 1994). One of these changes includes an alternation of the signal-processing in the vestibular system, specifically related to the changes in the gravity-sensitive otoliths. Whereas the otoliths still provide information about linear acceleration of the human body, they do not longer provide information about the orientation of the body relative to an external vertical reference. This leads to sensory conflict of the signals provided from the vestibular system and signals provided from other receptors and senses (e.g. vision, proprioception, sense of touch) which is assumed to cause a number of adverse effects like space motion sickness, disturbances of head-eye-coordination and gaze instability (Clement, 2011). In addition, the vestibular changes in space can also be expected to affect the efficiency of all basic or higher cognitive processes which usually depend on graviceptive cues, for example processes of spatial orientation, spatial perception and object recognition (Glasauer and Mittelstaedt 1998; Leone 1998; Oman 2007; see for a review Chap. 2).

Another system which is directly affected by the change of gravitational force in space is the sensorimotor system. Planning and execution of aimed voluntary movements involves a complex interaction of central motor programs responsible for sending efferent control signals to the effectors (e.g. arm, hand, fingers), and mechanisms of monitoring and adjusting ongoing movements based on afferent proprioceptive signals sent back from joints and muscles. Lacking the usual gravitational force in space can degrade both, the effectiveness of the central motor programs, as well as the processing of afferent feedback signals. All central motor programs which we use for certain classes of movements (e.g. pointing, grasping) have been acquired on Earth and, thus, have incorporated the gravitational force as an important component (Pozzo et al. 1998). This makes it possible to tune the force and timing of movements to the specific mechanical constraints provided 
by gravity. However, applying these same motor programs in space, can lead to inappropriate execution of movements which need to be corrected during execution. In addition, also the processing of afferent signals from joints and muscles needed to monitor and adjust movements if necessary, seems to be altered in microgravity which, in turn, can make online correction of movements more difficult and less effective in space than on Earth (Bock 1994). Analog to the sensory conflicts arising from the altered vestibular signals, the changes in the sensorimotor system have been assumed to cause a state of sensorimotor discordance (Bock 1998, p. 157), i.e. a disruption of the usual relationships between efferent and afferent signals used for the control of voluntary movements. This calls for a complex adaptation of movement control to the specifics of the space environment, including, for example, an adjustment of central motor programs, a re-weighting of afferent signals, and perhaps also a higher level of attentional control of movements. Although, these adaptive mechanisms are known to be highly effective, they can to lead to overt performance impairments in typical psychomotor tasks which will be described in some detail below.

The second class of stressors which can affect the cognitive and psychomotor performance of astronauts includes all stressors related to the typical working and living conditions in space. The majority of these stressor are not specific for spaceflight but include stressors which space habitats share with other sealed (technical) environments on Earth (capsule environments, Suedfeld \& Steel 2000). Examples of the latter include, e.g., submarines, stations for humans in Antarctica, or underwater habitats (Bishop 2011). Important social stressors of such isolated and confined environments (ICE) are social monotony and a lack of privacy which directly result from living within a small crew in a restricted living space. Environmental stressors in such habitats often include an elevated noise level and elevated levels of $\mathrm{CO} 2$ in the ambient air which both result from the technical requirements and constraints of life support systems necessary to support human life in such environments. For example, during working periods, the permanent noise level on the International Space Station (ISS) varies between 64 and $72 \mathrm{~dB}$, dependent on which module one stays in, and also noise levels during sleep periods were found to be higher (54-62 dB) than usually 
required for undisturbed sleep (Limardo et al. 2015). CO2 levels often increase up to $0.4-0.5 \%$ which is more than ten times higher as on Earth. Finally, also factors such as limited facilities for personal hygiene, need for extensive physical exercise on a daily basis, or permanent dependence on life support systems add to the stressors astronauts are exposed to aboard a space habitat. It can be assumed that a chronic exposure to such conglomerate of stressors can induce a stress state in astronauts which, in turn, may compromise their cognitive performance indicated, for example, by increased attentional selectivity, impairments of speed and/or accuracy of cognitive processes or impairments of working memory capacity (Hockey 1986).

\section{Impact of Spaceflight Related Stressors on Cognitive and Psychomotor Performance}

Results of two lines of research can be considered to assess the resilience of human cognitive and psychomotor performance during spaceflight. The first one includes specific neurocognitive experiments addressing the impact of microgravity on different perceptual, cognitive and psychomotor functions (Clement and Reschke 2008). These studies typically include controlled experiments during short-term spaceflights or the first days/weeks of long-term spaceflights which investigate performance in tasks where a direct impact of microgravity can be presumed. Among the main target areas of this research are tasks demanding spatial cognition or a precise control of voluntary movements. The second line of research includes what has been referred to as performance monitoring studies (Manzey 2000; Manzey and Lorenz 1998). Following a more general approach, these studies investigate the overall impact of living and working in a space habitat on human performance by repeatedly probing different cognitive and psychomotor functions of astronauts during a space mission. Performance assessments are usually conducted by standardized laboratory tasks which are well validated for this purpose. This enables for describing a whole pattern of performance changes over the course of a short-term or long-term space mission, although identifying the specific causes of these changes are often difficult to achieve. In the following, main insights of both lines of research with respect to the impact of spaceflight are described, structured according to different classes of performance functions. 


\subsection{Basic cognitive functions}

Basic cognitive functions, as understood here, include a wide variety of functions involved in choice reaction time tasks, visual search tasks, memory search tasks, mental arithmetic tasks, time estimation tasks, or logical reasoning tasks (e.g. grammatical reasoning). The impact of spaceflight on these functions has mainly been addressed by a number of performance monitoring studies mostly during short-term (Benke et al. 1993; Eddy et al. 1998; Kelly et al. 2005; Manzey et al. 1993; Newman and Lathan 1999; Ratino et al. 1988; Schiflett et al. 1995) but also long-term spaceflights (Manzey et al. 1998). It was assumed that these functions would perhaps not be affected by microgravity but that they might suffer from all the other space-relevant stressors astronauts are exposed to during a space mission (e.g. confinement, noise, social monotony). This expectation was based on findings that, on Earth, such stressors often induce a specific cognitive stress state in humans, reflected in specific impairments of attentional and cognitive processing (Hockey 1986). However, none of the performance monitoring studies referred to above has revealed any clear evidence for performance changes indicative for such effects during short-term spaceflight. A single-case performance monitoring study of an astronaut who has stayed for about 14 months on the former space station Mir suggest, that this is even true for extreme long stays in space (Manzey et al. 1998). In this latter study, basic cognitive functions were repeatedly probed by a grammatical reasoning task and two versions of a Sternberg memory-search task (Sternberg 1966). Performance was shown to remain stable on a level corresponding to the pre-flight baseline or even higher throughout the entire stay in space. Thus, it seems that basic cognitive functions remain largely resilient against the stress induced by the extreme living and working conditions in space.

\subsection{Spatial Cognition}

Spatial cognition includes different performance aspects which have mainly been addressed in a number of controlled spaceflight experiments. One of these aspects is related to processes involved in spatial orientation and navigation, i.e. the perception of the spatial position of the own body in relation to the ambient space. It has been shown that the lack of graviceptive cues during spaceflight 
usually lead to several distortions of spatial orientation, indicated, e.g., by erroneous perceptions of falling or rotating associated with head movements or sudden experiences of hanging upside down, at least during a transient period of adaptation to the space environment. However, it rarely causes a complete loss of the subjective feeling of up and down. Instead the majority of astronauts seems to keep such feeling related to the own body axis ("up is where the head is", Glasauer and Mittelstaedt 1998). These effects and related performance consequences in space have been reviewed elsewhere in some detail (Oman 2007; the chapter by Otmar Bock in this book) and shall not be repeated here.

Another aspect of spatial cognition includes processes of spatial imagery and object recognition.

These processes have been studied in a number of spaceflight experiments addressing the impact of microgravity on the perception of spatial relationships and the mental representation of threedimensional objects. One of the earliest studies investigated effects of the lack of graviceptive cues from the vestibular system on the assignment of spatial coordinates ("up", "down", "below", "above") to perceived objects (Friederici and Levelt 1990). They found that a consistent assignment of spatial coordinates is still possible in space. However, in contrast to Earth where such assignments are usually based on an unambiguous external frame of reference (e.g. information available from graviception and/or the visual structure of the ambient room), the assignments in space are based on an egocentric frame of reference provided by the retinal orientation of the perceived objects. This might impede any communication between astronauts about spatial relations unless the orientation of their body axes is aligned. However, other aspects of spatial processing and imagery seem to remain largely unchanged in space. For example, it is a well-known fact that, on Earth, humans have considerable difficulties to recognize objects that are presented in an unusual orientation. This has been shown in experiments where participants were presented simple figures (e.g. letters) in different orientations and were asked to decide as fast as possible whether they represented a normal or mirror-inversed version of the figure. A stable finding of such experiments is that decision times are the longer the more the presented figure deviates from its normal, i.e. upright, position (Shepard and Metzler 1971). This suggests that all sorts of spatial patterns and objects are cognitively 
represented in a certain orientation. If presented in a deviating orientation they first need to be "mentally rotated" in their normal position before they can be recognized. A set of studies has investigated whether these mental rotation processed were improved in microgravity (Clement et al. 1987; Leone et al. 1995a,b). This was expected because free-floating astronauts gain much more experiences than typical humans on Earth with seeing objects and other humans from unusual visual angles. However, the results of these studies suggest that the typical mental rotation effects known from Earth persist in space, also during long-term missions. This also holds true the so called face inversion effect, i.e. our difficulty to recognize even very familiar faces if presented in an inverted position. An experiment conducted on the former Mir station proved that astronauts are not better in identifying inversed faces in space than on ground (de Schonen et al. 1995). Still other aspects of spatial cognition have been assessed in a performance monitoring study during a short-term space mission (Benke et al. 1993). These aspects included memory for spatial patterns and spatial processing in a so called line judgment task. None of these performance aspects were found to be altered in space compared to a pre-flight baseline. Thus, overall, it seems that while processes of spatial orientation and navigation are considerably affected and compromised during spaceflight, at least during a transient period of adaptation to microgravity, other processes including spatial imagery, spatial memory or object recognition remain largely unaffected by the loss of graviceptive cues or the extreme working and living conditions during spaceflight.

\subsection{Psychomotor Functions}

Primarily two different classes of visuo-motor tasks have repeatedly used to investigate effects of spaceflight on psychomotor functions. The first class of tasks includes target-oriented movements with arm, hand and fingers, e.g. pointing at a given target, grasping a given object in the close environment, or positioning a joystick controlled cursor at a given target position on the screen, which need to be executed with or without visual feedback. Such sort of task has been used primarily in neuroscience studies in order to investigate the impact of microgravity on processes of (fine) motor control, reflected in changes of speed, accuracy and kinematics of such voluntary movements 
in space compared to executing these movements on ground (Berger et al., 1997; Bock et al., 2001; Jüngling and Bock 1999; Newman and Lathan 1999; Sangals et al. 1999; Watt 1997). The second class of movements includes continuous manual steering or tracking movements, e.g. compensating random movements of a cursor by proper counter movements at a joystick or following a moving target by a joystick-controlled cursor, and has been used in neuroscience and performance monitoring studies as well (Bock et al. 2001; Manzey et al. 1993, 1998; Schiflett et al. 1995). In contrast to discrete pointing or grasping movements which leave much degrees of freedom for movement planning and execution, tracking movements are more guided by an external cue which, e.g., pretends to move with a certain speed.

Converging results from research with both tasks suggest that psychomotor performance is considerably impaired in space at least for a transient period, suggesting a distortion of brain processes involved in visuo-motor transformations induced by the altered gravitational forces. However, the specific sorts of performance decrements differ between the two classes of movements. As has been shown repeatedly, discrete pointing or grasping movements with arm and fingers can be executed with the same precision in space than on Earth but only at the expense of a significantly slower movement time (Berger et al. 1997; Bock et al. 2001; Newman and Lathan 1999; Sangals et al. 1999). That is, astronauts are able to exactly point to given targets or grasp given objects in the ambient environment but need more time to do this in space compared to Earth. In contrast, a directly reversed effect often was found for tracking movements. Executing these movements with the required speed in space seems only be possible at the expense of precision, the latter reflected in a much larger tracking error or higher movement variability when performing such movements in space compared to Earth (Bock et al. 2001, 2010; Manzey et al. 1993, 1998, 2000). Altogether these results suggest that under the impact microgravity astronaut lose their capability to concurrently optimize both, accuracy and speed of voluntary movements (Bock et al. 2001).

The specific mechanisms leading to this reduced effectiveness of motor control in space are not sufficiently clear. One mechanism that has been proposed is an underestimation of the mass of 
extremities (arm, hand, fingers), due to the fact that weight is no longer a cue for mass under conditions of microgravity (Bock et al. 1996). That is, the perception of "weightless" extremities might have led to a miscalculation of forces needed to execute a certain movement. This fits nicely to findings from detailed analyses of the kinematics of directed movements with arm and fingers suggesting that movements are initiated with less acceleration in space than on Earth which then calls for additional adjustment processes during the execution of movements, resulting in a prolonged deceleration phase and longer movement time (Berger et al. 1997; Sangals et al. 1999). Based on this assumption one might expect that impairments of psychomotor performance would appear only for a short period after entering the microgravity environment and only the first executions of a certain psychomotor task in space. However, the few results available from longduration missions suggest that the described distortions of motor control processes can last for the first four weeks of space missions or even longer. During this period, at least tracking performance has been found to be particularly vulnerable to effects of fatigue, workload, and additional demands imposed by concurrent tasks (Bock et al. 2010; Manzey et al. 1998). This suggests that during adaptation to the space environment, the execution and monitoring of voluntary movements need more attentional effort in space than on Earth.

\subsection{Executive Functions}

Executive functions include all higher level cognitive processes which are involved in the cognitive control of goal-directed behaviour. Core sets of such functions include attentional and inhibitory control (e.g. selective attention and cognitive interference control, cognitive inhibition of impulses, old habits or response-sets), working memory (e.g. working with stored information), and cognitive flexibility (e.g. change of perspectives, divergent thinking) (Diamond 2014). Although all of these functions are highly significant for an effective control of behaviour, only very few studies have investigated the effectiveness of these functions during spaceflight, thus far. One set of studies has looked specifically at multitasking performance in space as an indicator of the effectiveness of executive function involved in attentional and inhibitory control. In these studies, astronauts were 
typically required to perform a psychomotor tasks (compensatory or pursuit tracking) task concurrently with a cognitive memory search task (Bock et al. 2010; Eddy et al. 1998; Fowler et al. 2000; Manzey et al. 1995, 1998; Schiflett et al. 1995). While some of these studies reported a dualtask performance decrement compared to baseline performance on Earth, (e.g. Manzey et al. 1995, 1998), others did not find a comparable performance decrement (Fowler et al. 2000) or attributed it to other factors than an impairment of executive functions (e.g. higher effort for motor programming; Bock et al. 2010). Another set of studies have used cognitive interference tasks (e.g. Stroop task) to probe the effectiveness of inhibitory control processes in space compared to Earth, and also found just a mixed pattern of results, with one study reporting no performance changes (Benke et al. 1993) and the other reporting impairments for a specific set of emotional and personal relevant stimuli (Pattyn et al. 2005). Finally, the speed to switch between two tasks was addressed in two performance monitoring studies during short-term spaceflights. Switching between two tasks involves a re-configuration of task sets which usually takes some time, reflected in so called switching costs, i.e., prolonged times to respond to a task after a switch as compared to response times for task repetitions (Monsell 2003). Comparing the performance of three (Schiflett et al. 1993) and four astronauts (Eddy et al. 1998) in space with their performance on ground, they found evidence for impaired switching performance in two out of the three and four subjects, respectively. Thus, overall, there is at least some evidence that executive functions and particular attentional and inhibitory control processes can become impaired in space compared to Earth. It does not seem very plausible that these functions suffer from the impact of altered gravitational force. More likely they reflect a general stress effect during short-term missions or the phase of primary adaptation during long-term missions. However, given the limited number of studies, the limited number of functions studied, and the somewhat inconsistent pattern of findings, any decisive conclusions about effects of the space environment on executive functions and higher cognitive processes can hardly be drawn at this time (Strangman et al. 2014).

\section{Summary and Conclusions}


The currently available evidence from spaceflight studies investigating effects of spaceflight on human cognitive and psychomotor performance suggests that at least basic perceptual and cognitive functions like visual search, memory search, grammatical reasoning, response selection, or time estimation remain largely intact and as efficient in space as on Earth. Given the large variety of environmental and social stressors present during spaceflight, it points to a remarkable resilience of these functions in astronauts. At least, astronauts seem to be able to compensate efficiently for possible impairments of such functions and prevent them from leading to overt performance decrements in simple cognitive tasks.

A different picture emerges for functions involved in spatial cognition, movement execution and control, and attentional control. Although astronauts are still able to perceive and assess spatial relationships in a consistent manner and keep a more or less strong feeling of up and down even in absence of the gravitational force, the frame of reference used for processing spatial relationship changes from an allocentric one to an egocentric one, the latter represented by the length axis of the own body. This has been shown to cause different perceptual illusions (e.g. visual reorientation illusions) and navigation problems within a space habitat which are described elsewhere in much more detail (Oman 2007; chapter by Otmar Bock in this book). However, processes of spatial imagery and object recognition, as assessed by mental rotation tasks, remain largely unchanged in space. Somewhat surprisingly astronauts are not even better to recognize objects seen from an unusual perspective. Obviously, the mental representation of objects remains orientation-dependent (i.e., they are mentally represented in an upright position) even with the lacking gravitational force in space. An example is the face inversion effect which persists in space. This is not only relevant for a better basic understanding of spatial cognition and object recognition but also of practical relevance. Specifically, it suggests that, for face-to-face communications with other crewmembers, astronauts should align their orientation in order to correctly perceive and interpret facial expressions which provide important non-verbal cues for interpersonal communication (Cohen 2000). 
Sensorimotor processes involved in planning and execution of voluntary movements seem to be significantly disturbed at least during a transient period of adaptation to space which can involve the first three to four weeks of a space mission. During this period, the execution of movements often takes longer or lacks precision, dependent on the priority of these two aspects. Due to the microgravity-induced changes in the sensorimotor system, the execution of precise voluntary movements seems to become more attention demanding than on Earth, and it becomes more difficult to optimize effectiveness (accuracy) and efficiency (speed) of movements at the same time. This has direct consequences for the planning and scheduling of missions tasks for astronauts. At least during the first four weeks of a space mission it needs to be taken into account that astronauts may need considerable more time in space than during pre-flight training on Earth to perform certain mission tasks effectively. Even though they might be able to prevent obvious performance decrements by investing additional effort, their psychomotor performance can remain vulnerable to effects of fatigue, workload and stress until their sensorimotor system have fully adapt to the altered gravitational force in space.

Last but not least, also higher executive functions of attentional control seem to be somewhat degraded during spaceflight. However, the current evidence is somewhat weak and contradictory, and the specific origins of these effects are not fully clear, yet.

A general limitation of our current knowledge about cognitive and psychomotor performance is that most of it has only been gained from research during short-term spaceflights. Only very few studies have actually been conducted during long-term space missions lasting longer than three months, and only one single-case study is available, thus far, which has addressed performance of one astronaut whose continuous time in space came close in duration to future missions to Mars (i.e. 14 months; Manzey et al. 1998). The results of this latter study prove that at least this individual cosmonaut was able to maintain his cognitive performance on a comparatively high level, even across a extreme long time of living and working in a space habitat. His psychomotor performance just showed impairments and some variability for about the first four weeks in space. Than it also returned to the 
pre-flight baseline level and stayed constant across the entire mission. However, it is not yet clear how representative these findings are. Recent results from a ground-based simulation of a 500 days Mars mission provide evidence that individuals can largely differ with respect to their behavioural adaptation to long-term confinement and isolation (Basner et al. 2014). Certainly more research from missions lasting one year or longer is needed before the possible risks of human performance decrements associated with future exploratory space flights can eventually be assessed.

\section{References}

Basner M, Dinges DF, Mollicone DJ, Savelev I, Ecker AJ, Di Antonio A, Jones CW, Hyder EC, Kan K, Morukov BV, Sutton JP (2014) Psychological and behavioral changes during confinement in a 520-day simulated interplanetary mission to Mars. PLoS ONE 9: e93298. doi:10.1371/journal.pone.0093298.

Benke T, Koserenko O, Watson NV, Gerstenbrand F (1993) Space and cognition: The measurement of behavioral functions during a 6-day space mission. Aviat Space Environ Med 64:376-79.

Berger M, Mescheriakov S, Molokanova E, Lechner-Steinleitner S, Seguer N, Kozlovskaya V (1997) Pointing arm movements in short- and long-term spaceflight. Aviat Space Environ Med 68:781-787.

Bishop SL (2011) From Earth analogs to space: Getting there from here. In: Vakoch TA (ed) Psychology of space exploration. Contemporary Research in Historical Perspective. NASA SP-20114411. Nasa, Washington, p. 47-78.

Bock O (1998) Problems of sensorimotor coordination in weightlessness. Brain Res Rev 28:155-160. Bock O (1994) Joint position sense in simulated changed-gravity environments. Aviat Space Environ Med 65:621-626.

Bock O, Arnold KE, Cheung BSK (1996) Performance of a simple aiming task in hypergravity: II. Detailed response characteristics. Aviat Space Environ Med 67:133-138. 
Bock O, Fowler B, Comfort D (2001) Human sensorimotor coordination during spaceflight: An analysis of pointing and tracking responses during the "Neurolab" space shuttle mission. Aviat Space Environ Med 72:877-883.

Bock O, Howard IP, Money KE Arnold KE (1992) Accuracy of aimed arm movements in changed gravity. Aviat Space Environ Med 63:994-998.

Bock O, Weigelt C, Bloomberg JJ (2010) Cognitive demand of human sensorimotor performance during an extended space-mission: a dual-task study. Aviat Space Environ Med 81: 819-824.

Clement G (2011) Fundamentals of space medicine. 2nd edition. Heidelberg: Springer.

Clement G, Reschke MF (2008) Neuroscience in space. Heidelberg: Springer.

Clément G, Berthoz A, Lestienne F (1987) Adaptive changes in perception of body orientation and mental image rotation in microgravity. Aviat Space Environ Med 58:A159-163.

Cruse H, Dean J, Heuer H, Schmidt, RA (1990) Utilization of sensory information for motor control. In: Neumann O, Prinz W (eds) Relationships Between Perception and Action. Springer, Heidelberg, p... de Schonen S, Leone G, Lipshits M (1998) The face inversion effect in microgravity: is gravity used as a spatial reference for complex object recognition ? Acta Astronautica 42:287-301.

Diamond A (2014) Executive functions. Annu Rev Psychol 64: 135-168.

Eddy D, Schiflett S, Schlegel R, Shehab R (1998) Cognitive performance aboard the life and microgravity spacelab. Acta Astronautica 43:193-210.

Ellis SR (2000) Collision in space. Ergonomics in Design 8:4-9.

Fowler B, Bock O, Comfort D (2000). Is dual-task performance necessarily impaired in space ? Human Factors 42:318-326.

Friederici AD, Levelt WJM (1990) Spatial reference in weightlessness: perceptual factors and mental representations. Perc Psychophy 47:253-266. 
Garriott OK, Doerre GL (1977) Crew efficiency on first exposure to zero-gravity. In: Johnston RS, Dietlein LF (eds) Biomedical results from Skylab (NASA-SP377). Nasa, Washington.

Glasauer S, Mittelstaedt H (1998) Perception of spatial orientation in microgravity. Brain Res Rev 28:185-193.

Heuer H, Manzey D, Lorenz B, Sangals J (2003) Impairments of manual tracking performance during spaceflight are associated with specific effects of microgravity on visuomotor transformations. Ergonomics 46: 920-934.

Hockey GRJ (1986) Changes in operator efficiency as a function of environmental stress, fatigue, and circadian rhythms. In: Boff KR, Kaufman L, Thomas JP (eds) Handbook of Perception and Performance. Vol II: Cognitive Processes and Performance. Wiley, New York, p 44-1 - 44-49 .

Jüngling S, Bock O (1999) Sensorimotor adaptation of humans to the space environment. Proceedings of the 2nd European Symposium on the Utilisation of the International Space Station (ESA SP-433). ESA, Noordwijk, p. 527-531.

Kelly TH, Hienz RD, Zarcone, TJ, Wurster RM, Brady JV (2005) Crewmember performance before, during, and after spaceflight. J Exp Anal Beh 84: 227-241.

Kornilova, LN (1997) Orientation illusions in space flight. J Vest Res 7:429-439.

Leone G (1998) The effect of gravity on human recognition of disoriented objects. Brain Res Rev 28:203-214.

Leone G, Lipshits M, Gurfinkel V, Berthoz A (1995a) Influence of graviceptive cues at different levels of visual information processing: The effects of prolonged weightlessness. Acta Astronautica 36:743751.

Leone G, Lipshits M, Gurfinkel V, Berthoz A (1995b) Is there an effect of weightlessness on mental rotation of three-dimensional objects ? Cog Brain Res 2:255-267. 
Limardo JG, Allen CS, Danielson RW (2015) Status: Crew member noise exposures on the International Space Station. Proceedings of the 45th International Conference on Environmental Systems, 12-16 July 2015, Bellevue, Washington. [available from: https://ntrs.nasa.gov/archive/nasa/ casi.ntrs.nasa.gov/20150011048.pdf; last access: 06 Nov 2016].

Manzey D (2000) Monitoring of mental performance during spaceflight. Aviat Space Environ Med 71:A69-75.

Manzey D, Kanas N (2008). Space psychology and psychiatry. Springer, Heidelberg.

Manzey D, Lorenz B (1998a) Mental performance during short-term and long-term spaceflight. Brain Res Rev 28:215-21.

Manzey D, Lorenz B, Heuer H, Sangals J (2000) Impairments of manual tracking performance in space: More converging evidence from a 20-day space mission. Ergonomics 43: 589-609.

Manzey D, Lorenz B, Polyakov VV (1998) Mental performance in extreme environments: Results from a performance monitoring study during a 438-day space mission. Ergonomics 41:537-59.

Manzey D, Lorenz B, Schiewe A, Finell G, Thiele G (1993) Behavioral aspects of human adaptation to space: Analyses of cognitive and psychomotor performance during an 8-days space mission. Clin Inv 71:725-31.

Manzey D, Lorenz B, Schiewe A, Finell G, Thiele G (1995) Dual-task performance in space: Results from a single-case study during a short-term space mission. Hum Fac 37:667-81.

Monsell (2003) Task switching. Trends in Cognitive Sciences 7: 134-140.

Morphew E, Balmer DV, Khoury GJ (2001) Human performance in space. Ergonomics in Design 9: 611.

Newberg AB (1994) Changes in the central nervous system and their clinical correlates during longterm space flight. Aviat Space Environ Med 65:562-572. 
Newman DJ, Lathan, CE (1999) Memory processes and motor control in extreme environments. IEEE Trans Sys Man Cyber: Part C: Appl Rev 29:387-394.

Oman, CM (2007) Spatial orientation and navigation in microgravity. In Mast F, Jancke L (eds) Spatial processing in navigation, imagery and perception. Springer, Heidelberg, p 209-247

Pozzo T, Papaxanthis C, Stapley P, Berthoz A (1998) The sensorimotor and cognitive integration of gravity. Brain Res Rev 28:92-101.

Ratino DA, Repperger DW, Goodyear C, Potor G, Rodriguez LE (1988) Quantification of reaction time and time perception during Space Shuttle operations. Aviat Space Environ Med 59:220-24

Sangals J, Heuer H, Manzey D, Lorenz B (1999) Changed visuomotor transformations during and after spaceflight. Exp Brain Res 129: 378-390.

Schiflett S, Eddy D, Schlegel RE, French J, Shehab R (1995) Performance Assessment Workstation (PAWS). Unpublished Final Science Report to NASA.

Shepard RN, Metzler J (1971) Mental rotation of three-dimensional objects. Science 171: 701-703.

Sternberg S (1966) High-speed scanning in human memory. Science 153: 652-654.

Strangman et al. (2014) Human cognitive performance in spaceflight and analog environments. Aviat Space Environ Med 85:1033-48.

Suedfeld P, Steel GD (2000) The environmental psychology of capsule habitats. Ann Rev of Psych 51: 227-53.

Watt DGD (1997) Pointing at memorized targets during prolonged microgravity. Aviat Space Environ Med 68:99-103. 